Originales

\title{
Análisis de las variables sociosanitarias asociadas a la permanencia en incapacidad temporal
}

\author{
Analyses of the sociosanitary variables associated with the permanency in \\ temporary disability
}

M. ${ }^{a}$ Luz Pérez Morote', Jesús López-Torres Hidalgo², M. ${ }^{a}$ Ángeles López Verdejo ${ }^{2}$

1. Unidad Médica del Equipo de Valoración de Incapacidades. Dirección Provincial INSS Albacete. España.

2. Gerencia de Atención Integrada de Albacete (Servicio de Salud de Castilla-La Mancha) y Facultad de Medicina de Albacete (Universidad de Castilla la Mancha). Albacete. España.

Recibido: 16-12-14

Aceptado: 08-02-15

\section{Correspondencia}

M. ${ }^{a}$ Luz Pérez Morote

C/ Lérida, n. ${ }^{\circ} 24,4{ }^{\circ} \mathrm{A}$

(02006) Albacete. España.

Teléfono: 616024168

Correo electrónico: mluzpm@yahoo.es

ResUmen

Introducción: La incapacidad temporal (IT) constituye un proceso de origen multifactorial.

Objetivo: Evaluar el proceso de IT en relación con la enfermedad, estado de salud, satisfacción laboral, perfil de locus de control y características sociodemográficas.

Método: Estudio observacional de cohortes prospectivo sobre 404 pacientes en IT. Realizamos un análisis de supervivencia describiendo la evolución de los participantes en cuanto a su permanencia en IT.

Resultados: La permanencia media en IT fue significativamente superior $(\mathrm{p}<0,05)$ en sujetos con nivel de instrucción bajo (9,4 meses), trabajadores manuales (8,5 meses), mayores de 60 años (10,0 meses), pacientes insatisfechos laboralmente (9,3 meses), con baja autopercepción de salud (10,2 meses), consumidores de medicación crónica (10,6 meses), fumadores (9,3 meses) y consumidores de drogas no institucionalizadas (10,5 meses). Tras el análisis multivariante, se asocian a una menor duración de la IT: las enfermedades del aparato locomotor, sistema cardiorrespiratorio o problemas psicológicos, un nivel de instrucción alto, una buena autopercepción de salud y la abstención en el consumo de tóxicos.

Conclusiones: Además de la enfermedad, otros factores relacionados con la percepción del estado de salud, características sociodemográficas y el estilo de vida, influyen de manera significativa en la reincorporación de un trabajador a su actividad laboral.

Med Segur Trab (Internet) 2015; 61 (239) 184-194

Palabras clave: Absentismo laboral; incapacidad profesional; salud laboral. 


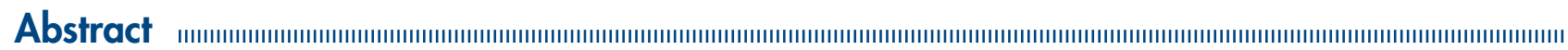

Introduction: The temporary disability (IT) refers to a process of multifactorial origin.

AIM: To evaluate this process in relation with the corresponding disease, health status, labor satisfaction, locus of control profile and socio-demographic characteristics.

Methods: A prospective observational cohort study was performed on 404 patients in IT. We carried out an analysis of survival describing the evolution of the participants as well as their permanency in IT.

Results: The permanency average in IT was significantly high $(\mathrm{p}<0,05)$ in subjects with a lower level of instruction (9,4 months), manual workers (8,5 months), over 60 years old (10,0 months), labor unsatisfied patients (9,3 months), with low health self-awareness (10,2 months), consumers of chronic medication (10,6 months), smokers (9,3 months) and consumers of not institutionalized drugs (10,5 months). After the multivariate analysis, they associate the diseases of the locomotive device, the cardiorespiratory system or psychological problems, a high level of instruction, a good auto perception of health and the abstention in the consumption of toxins to a minor duration of the IT.

Conclusions: Besides the disease, other factors related to the perception of the health status, sociodemographic characteristics and the way of life, influence in a significant way the reincorporation of a worker to his labor activity.

Med Segur Trab (Internet) 2015; 61 (239) 184-194

Keywords: labor absenteeism; professional disability; labor bealth. 


\section{INTRODUCCIÓN}

En el año 2014 la Seguridad Social gestionó el 37,1 \% del gasto de los presupuestos generales del Estado, siendo el presupuesto inicial destinado para la prestación de incapacidad temporal (IT) de 4.878, 35 millones de euros. El gasto en prestación de IT supuso el doble que el gasto en prestaciones no contributivas y el triple que el gasto en prestaciones familiares ${ }^{1}$.

El gasto en IT no viene solo determinado por el número de procesos de baja que se tramitan, sino también por la duración de los mismos. Algunos estudios estiman que un $20 \%$ de las incapacidades temporales duran más tiempo de lo que el médico que las prescribe opina que deberían haber durado conforme al proceso clínico del paciente ${ }^{2}$.

Entre las causas que se muestran para justificar éste incremento en la duración de la IT se incluyen las demoras que genera la propia organización sanitaria, la utilización indebida de ésta prestación para dar cobertura a otras carencias del sistema de protección social, o bien la coexistencia de procesos intercurrentes que prolongan la situación de IT más allá de lo esperado inicialmente.

La correcta gestión de la prestación de IT debe realizarse tomando como punto de partida el origen multifactorial de la misma y así dicha gestión pasa por un conocimiento preciso de los factores pronósticos de la duración que sean modificables mediante intervenciones asistenciales y laborales.

Para un trabajador la vuelta a su puesto de trabajo después de una situación de IT no sólo viene determinada por la naturaleza de la enfermedad que motivó el inicio de la baja, sino que ésta puede verse influenciada por una serie de factores ${ }^{3}$ : a) sociodemográficos, como edad $^{4-6}, \operatorname{sexo}^{7,8}$ o estado civil ${ }^{9,10}$; b) laborales, como categoría profesional ${ }^{11,12}$, antigüedad profesional, tipo de trabajo, grado de satisfacción laboral ${ }^{13}$ o tipo de contrato $^{14}$; y c) médicos, como el tipo de enfermedad ${ }^{15}$.

En España, el inicio de la IT viene determinada por la indicación del médico de atención primaria, siendo éste en la mayoría de los casos quien decide el momento de la reincorporación laboral. Sin embargo, no existen actualmente criterios definidos para determinar la duración de la incapacidad temporal, pese a que algunos autores han determinado valores de referencia y sugieren la realización de otros estudios que continúen esta labor ${ }^{16}$.

A pesar de que en los últimos años se observa una progresiva reducción del gasto en prestaciones de IT, debido por un lado a la reducción de la población protegida, a la menor incidencia de la misma y a la eficacia de las medidas de control, surge la necesidad de seguir trabajando en la búsqueda de herramientas de gestión útiles para controlar este gasto.

El objetivo de éste estudio es evaluar el resultado de los procesos prolongados de IT de los trabajadores en relación con la enfermedad y el estado de salud, la satisfacción laboral, la autopercepción de salud, el perfil de locus de control y las circunstancias sociodemográficas, evaluando si dichas variables condicionan o no la permanencia en IT.

\section{MATERIAL Y MÉTODOS}

Se trata de un estudio observacional de cohortes prospectivo en el que una muestra de 404 pacientes en situación de IT, pertenecientes al Área Sanitaria de Albacete, ha sido evaluada al tercer mes de iniciada la prestación en la Unidad Médica del Equipo de Valoración de Incapacidades (UMEVI), efectuando posteriormente un seguimiento hasta cumplirse un periodo de 12 meses desde el inicio de dicha incapacidad. Los pacientes fueron seleccionados consecutivamente durante el periodo comprendido entre los meses de agosto de 2009 y octubre de 2011. 
En todos los casos seleccionados los criterios de inclusión fueron:

Pacientes revisados por primera vez en la UMEVI para el control de su periodo de IT.

Pacientes en cuya revisión fue determinada la adecuación de la permanencia en IT.

Pacientes cuyo estado de salud físico o mental permitió la valoración en la UMEVI sin necesidad de desplazamiento al domicilio o a un centro sanitario.

Los criterios tenidos en cuenta para excluir a los pacientes del estudio fueron:

Pacientes que tras ser citados para control de su periodo de IT por primera vez en la UMEVI fueron dados de alta por parte del médico inspector ese mismo día.

Mujeres en situación de IT por causa de embarazo.

Pacientes ingresados o encamados que no pudieron desplazarse a la UMEVI para su valoración.

En todos los casos seleccionados se determinó al cabo de un año del inicio del proceso de IT cuál fue el motivo de resolución del mismo.

El constructo locus de control, hace referencia a las expectativas sobre relación entre la conducta y sus resultados o consecuencias. Se define por la creencia del sujeto en la responsabilidad que tiene sobre su propio actuar. Se evaluó mediante la escala de «juicios de control sobre los agentes de salud", diseñada por Linares (2001) ${ }^{17}$. El grado de satisfacción laboral se evaluó mediante la Escala General de Satisfacción ${ }^{18}$. La autopercepción de salud se evaluó con la pregunta: "En los últimos meses, ¿diría usted que su estado de salud es muy bueno, bueno, regular, malo o muy malo?». La variable "Categoría social"se midió utilizando la clasificación de Goldthorpe ${ }^{19}$. Se recogieron datos sobre variables relacionadas con el estado de salud (diagnóstico principal de la baja según la Clasificación Internacional en Atención Primaria, CIAP-2; antecedentes de enfermedad mental según el epígrafe de "problemas psicológicos" de la CIAP-2; consumo de medicación crónica y hábitos tóxicos), variables sociodemográficas (sexo, edad, estado civil y nivel de instrucción), antecedentes de IT previa y posesión o no de una incapacidad permanente.

El estudio se adhiere por completo a las Normas de Buena Práctica Clínica y a los principios básicos establecidos en la Declaración de Helsinki. Se contó con la autorización de la Dirección General del INSS para poder acceder a los datos laborales de los pacientes y el estudio fue aprobado por el Comité Ético de Investigación Clínica de la Gerencia de Atención Integrada de Albacete.

En cuanto al análisis estadístico se realizó un análisis de supervivencia para describir la evolución de los sujetos en cuanto a su permanencia en IT, calculando la probabilidad de dicha permanencia en diferentes intervalos de tiempo. Como técnica de análisis se utilizó el método de estimación actuarial. La variable «tiempo de permanencia en IT» fue obtenida a partir de la fecha de inicio del periodo de IT y de la fecha en la que se emitió el alta laboral. En cada caso se calculó la mediana del tiempo de supervivencia. Posteriormente, se procedió al cálculo de las curvas de supervivencia por el método de Kaplan-Meier, representando en función del tiempo las probabilidades acumuladas de supervivencia estimadas (probabilidad de que un paciente permanezca en IT en cada periodo considerado). Se realizaron comparaciones de curvas de supervivencia mediante la prueba de Mantel-Haenszel (Log Rank), que permitió comparar las altas laborales observadas en diferentes grupos con las esperadas en caso de no existir diferencia (ley de ji-cuadrado).

Finalmente, se construyó un modelo de riesgos proporcionales de Cox para analizar los efectos de las variables independientes consideradas sobre la variable dependiente "tiempo de permanencia en IT». Dicho modelo permitió establecer factores pronósticos significativamente relacionados con el tiempo de permanencia en IT. 


\section{RESULTADOS}

Transcurridos 12 meses desde el inicio de la IT, pudo observarse que las causas más frecuentes de alta fueron el «alta por mejoría» emitida por el médico de familia con anterioridad al agotamiento del periodo de IT (43,6\%), la solicitud de prórroga del proceso de IT $(15,1 \%)$ y el alta a propuesta del médico inspector $(12,4 \%)$.

Los 12 meses fueron agotados por 164 pacientes $(40,6 \%)$ y la duración media de los procesos de IT fue de 260,4 días (DE: 94,2; IC 95\%: 251,1 - 269,6), con una mediana de 263,5 días (rango intercuartil: 186,5 días). Del total de pacientes, el 59,4\% (240 pacientes) recibieron el alta médica antes de agotar el periodo de 12 meses con la finalidad de reincorporarse a su trabajo.

En la tabla 1 y en la figura 1 se muestra la probabilidad acumulada de permanencia en IT en diferentes intervalos de tiempo (meses).

Tabla 1. Probabilidad acumulada de permanencia en incapacidad temporal en diferentes meses

\begin{tabular}{|c|c|c|c|c|c|c|}
\hline $\begin{array}{l}\text { Intervalo } \\
\text { de tiempo } \\
\text { (meses) }\end{array}$ & N. ${ }^{\circ}$ sujetos & N. ${ }^{\circ}$ altas & $\begin{array}{l}\text { Proporción } \\
\text { acumulada que } \\
\text { permanece en } \\
\text { IT (PA) }\end{array}$ & $\begin{array}{l}\text { Error estándar } \\
\text { (PA) }\end{array}$ & $\begin{array}{c}\text { Densidad de } \\
\text { probabilidad } \\
\text { (DP) }\end{array}$ & $\begin{array}{l}\text { Error estándar } \\
\text { (DP) }\end{array}$ \\
\hline $1 .^{\circ}$ & 404 & 1 & 1,00 & 0,00 & 0,002 & 0,002 \\
\hline $2 .^{\circ}$ & 400 & 0 & 1,00 & 0,00 & 0,000 & 0,000 \\
\hline $3 .^{\circ}$ & 400 & 2 & 0,99 & 0,00 & 0,005 & 0,004 \\
\hline $4 .^{\circ}$ & 398 & 14 & 0,96 & 0,01 & 0,035 & 0,009 \\
\hline $5 .^{\circ}$ & 382 & 40 & 0,86 & 0,02 & 0,101 & 0,015 \\
\hline $6 .^{\circ}$ & 338 & 34 & 0,77 & 0,02 & 0,087 & 0,014 \\
\hline $7 .^{\circ}$ & 300 & 31 & 0,69 & 0,02 & 0,080 & 0,014 \\
\hline $8 .^{\circ}$ & 265 & 27 & 0,62 & 0,02 & 0,071 & 0,013 \\
\hline $9 .^{\circ}$ & 235 & 33 & 0,53 & 0,03 & 0,088 & 0,015 \\
\hline $10 .^{\circ}$ & 195 & 23 & 0,47 & 0,03 & 0,063 & 0,013 \\
\hline $11 .^{\circ}$ & 167 & 21 & 0,41 & 0,03 & 0,059 & 0,012 \\
\hline $12 .^{\circ}$ & 144 & 64 & 0,16 & 0,02 & 0,251 & 0,025 \\
\hline
\end{tabular}

Figura 1. Representación gráfica de la probabilidad acumulada de permanencia en incapacidad temporal en diferentes meses

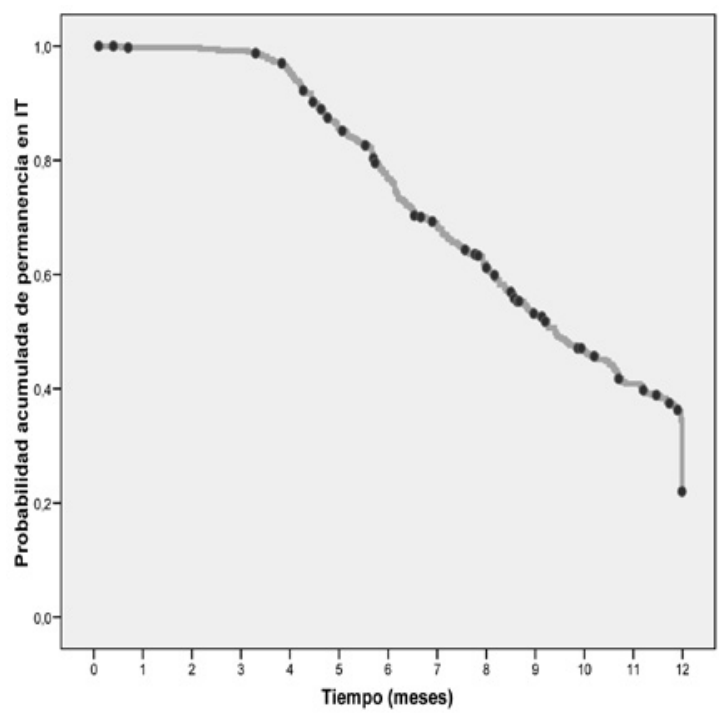


La permanencia en IT fue significativamente superior en (tabla 2): sujetos con sólo estudios primarios o sin estudios, trabajadores manuales (categorías sociales IIIc a V), mayores de 60 años, menos satisfechos laboralmente (puntuación inferior al percentil 50 de la distribución), con peor autopercepción de salud, polimedicados, fumadores y consumidores de drogas no institucionalizadas. En relación al perfil del locus de control de los participantes y la permanencia en IT de los mismos, no se obtuvieron diferencias estadísticamente significativas al comparar los locus «azar», «externo», «indeterminado»e «interno».

Tabla 2. Permanencia en IT de los participantes según diferentes variables

\begin{tabular}{|c|c|c|c|}
\hline Variables & $\begin{array}{l}\text { Permanencia media } \\
\text { en IT (IC 95\%) }\end{array}$ & Mediana (IC 95\%) & $\mathbf{p}$ \\
\hline \multicolumn{4}{|l|}{ Nivel de instrucción: } \\
\hline - Al menos enseñanza secundaria & $8,3(8,0-8,8)$ & $8,2(7,3-9,1)$ & 0,003 \\
\hline - Estudios primarios o sin estudios & $9,4(9,0-9,8)$ & $10,6(9,3-12,0)$ & \\
\hline \multicolumn{4}{|l|}{ Categoría social: } \\
\hline - I a IIIb & $8,5(8,1-9,0)$ & $8,5(7,9-9,2)$ & 0,03 \\
\hline - IIIc a V & $9,0(8,6-9,2)$ & $10,5(9,2-11,7)$ & \\
\hline \multicolumn{4}{|l|}{ Edad: } \\
\hline - Mayores de 60 años & $10,0(9,4-10,6)$ & $11,2(10,6-11,7)$ & 0,01 \\
\hline - Menores de 60 años & $8,7(8,4-9,0)$ & $9,0(8,2-9,6)$ & \\
\hline \multicolumn{4}{|l|}{ Satisfacción laboral: } \\
\hline - Menos satisfechos & $9,3(8,8-9,7)$ & $8,6(8,1-9,0)$ & 0,03 \\
\hline - Más satisfechos & $10,5(9,3-11,6)$ & $8,5(7,7-9,3)$ & \\
\hline \multicolumn{4}{|l|}{ Autopercepción de salud: } \\
\hline - Buena/muy buena & $8,5(8,1-8,9)$ & $8,5(7,8-9,2)$ & 0,004 \\
\hline - Regular & $9,4(9,0-10,0)$ & $10,6(9,6-11,6)$ & \\
\hline - Mala/muy mala & $10,2(8,6-9,2)$ & $9,4(8,5-10,2)$ & \\
\hline \multicolumn{4}{|l|}{ Consumo de medicación crónica: } \\
\hline - 5 o más fármacos & $9,4(8,6-10,3)$ & $10,5(7,3-13,7)$ & 0,02 \\
\hline - 1 - 4 fármacos & $9,5(9,0-9,9)$ & $10,6(9,8-11,4)$ & \\
\hline - No consumo & $8,3(7,8-8,7)$ & $8,0(7,1-9,0)$ & \\
\hline \multicolumn{4}{|l|}{ Consumo de tabaco: } \\
\hline - Fumadores & $9,3(8,9-9,8)$ & $10,8(9,5-12,0)$ & 0,02 \\
\hline - No fumadores & $8,7(8,3-9,1)$ & $8,6(8,0-9,3)$ & \\
\hline \multicolumn{4}{|l|}{$\begin{array}{l}\text { Consumo de drogas no } \\
\text { institucionalizadas: }\end{array}$} \\
\hline - Sí & $10,5(9,1-11,8)$ & $8.5(7,7-9,3)$ & 0,01 \\
\hline - No & $8,9(8,6-9,2)$ & $8,8(8,1-9,4)$ & \\
\hline
\end{tabular}

Al analizar la permanencia en IT de los sujetos estudiados en relación con su enfermedad se obtuvieron los siguientes resultados:

- Enfermedades del aparato locomotor: permanencia media en IT 8,8 meses (IC 95\%: 8,3-9,2); mediana 8,9 meses (IC 95\%: 7,9-9,9).

- Trastornos psicológicos: permanencia media en IT 8,8 meses (IC 95\%: 8,2-9,5); mediana 9,3 meses (IC 95\%: 8,2-10,4).

- Enfermedades cardiorrespiratorias: permanencia media en IT 9,0 meses (IC 95\%: 8,4-9,6); mediana 10,1 meses (IC 95\%: 8,4-11,8). 
- Otras enfermedades: permanencia media en IT 9,9 meses (IC 95\%: 9,0-10,8); mediana 11,8 meses (IC 95\%: 10,6-13,1).

Aunque los resultados fueron diferentes en los grupos anteriores, resultando superior la permanencia en IT en los sujetos clasificados como "otras enfermedades», únicamente se alcanzó una diferencia estadísticamente significativa entre éstos y los que presentaban enfermedades del aparato locomotor $(p=0,02)$.

En el análisis de regresión de Cox efectuado para valorar el efecto de las diferentes variables de estudio sobre la permanencia en IT, las variables asociadas a una menor duración de ésta que permanecieron en el modelo fueron las siguientes: enfermedades del aparato locomotor, sistema cardiorrespiratorio y problemas psicológicos; mayor nivel de instrucción (al menos enseñanza secundaria); mejor autopercepción de salud (calificada como buena o muy buena); abstención en el consumo de tóxicos diferentes a alcohol o tabaco. Como se muestra en la tabla 3, en el caso de las enfermedades del aparato locomotor, sistema cardiorrespiratorio y problemas psicológicos la finalización del periodo de IT se multiplica por 1,61 (HR = 1,608; IC 95\%: 1,038 - 2,492; p = 0,03), en los sujetos con mayor nivel de instrucción (al menos enseñanza secundaria) se multiplica por 1,42 (HR = 1,419; IC 95\%: 1,125 - 1,791; $\mathrm{p}=0,003)$, en quienes perciben su salud como buena o muy buena se multiplica por 1,31 (HR = 1,310; IC 95\%: 1,029 - 1,668; p = 0,02) $\mathrm{y}$ en quienes se abstienen del consumo de tóxicos diferentes a alcohol o tabaco se multiplica por 2,44 (HR = 2,442; IC 95\%: 1,077 - 5,537; $\mathrm{p}=0,03)$. En la figura 2 se muestra la representación gráfica de las variables asociadas a la permanencia en IT mediante un modelo de regresión de Cox.

Tabla 3. Variables asociadas mediante regresión de Cox a la permanencia en incapacidad temporal. HR: Hazard Ratio

\begin{tabular}{lcccc}
\hline \multicolumn{1}{c}{ Variables } & Coeficiente & HR & IC 95\% & p \\
\hline $\begin{array}{l}\text { Enfermedades del aparato locomotor, sistema } \\
\text { cardiorrespiratorio y problemas psicológicos }\end{array}$ & 0,475 & 1,608 & $1,038-2,492$ & 0,03 \\
$\begin{array}{l}\text { Mayor nivel de instrucción (al menos } \\
\text { enseñanza secundaria). }\end{array}$ & 0,35 & 1,419 & $1,125-1,791$ & 0,003 \\
$\begin{array}{l}\text { Mayor autopercepción de salud (calificada } \\
\text { como buena o muy buena) }\end{array}$ & 0,27 & 1,310 & $1,029-1,668$ & 0,02 \\
$\begin{array}{l}\text { Abstención en el consumo de tóxicos } \\
\text { diferentes a alcohol o tabaco }\end{array}$ & 0,893 & 2,442 & $1,077-5,537$ & 0,03 \\
\hline
\end{tabular}

Figura 2. Representación gráfica de las variables asociadas a la permanencia en incapacidad temporal mediante un modelo de regresión de Cox. AL: Aparato Locomotor, PS Problemas Psicológicos, SCR: Sistema Cardiorrespiratorio

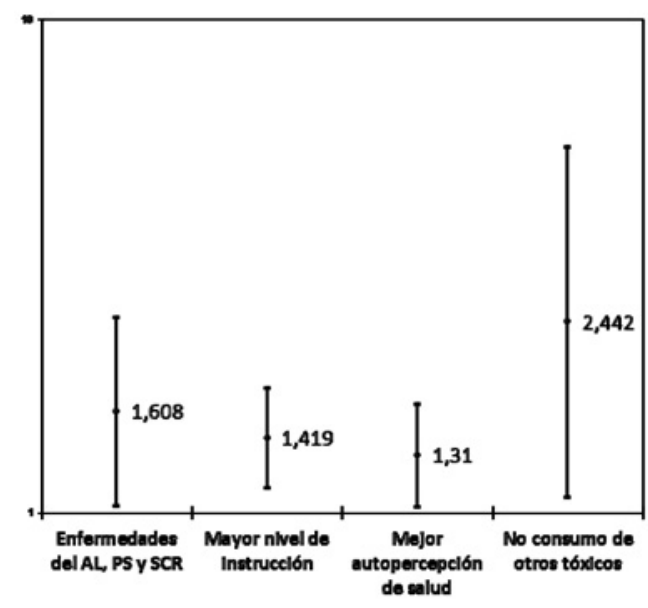




\section{DISCUSIÓN}

Nuestro estudio demuestra que, además de la enfermedad padecida por el paciente, otros factores tales como el nivel de instrucción, el nivel de salud autopercibida y el consumo de tóxicos influyen de manera significativa en la reincorporación de un trabajador a su actividad laboral. Estos factores, que contribuyen independientemente en la resolución del proceso de IT, indican que éste constituye una situación multifactorial en la que intervienen aspectos relacionados con la salud, la situación sociodemográfica y los estilos de vida.

En la valoración de la metodología empleada para la realización de este estudio, la principal limitación reside en que la información que se puede obtener de la descripción de la IT no es representativa del total de la misma, dado que no se han recogido las bajas de corta duración. Como otra limitación de nuestro estudio debe mencionarse que los datos analizados en relación con la evolución del periodo de IT se circunscriben a una única área sanitaria, lo cual puede suponer una dificultad a la hora de extrapolar los resultados a otras poblaciones con diferentes características sociodemográficas, culturales o educativas. Por otra parte, la muestra contempla situaciones de IT únicamente por contingencia común (ITcc), dejando al margen aquéllos procesos de IT derivados de contingencias profesionales (ITcp) cuya gestión es llevada a cabo por las MATEPSS. No obstante, la selección de los pacientes se ha realizado de forma consecutiva a lo largo de diferentes años hasta alcanzar un tamaño muestral suficiente para el estudio, pudiendo considerarse la muestra representativa de todos los sujetos susceptibles de ser estudiados.

En relación con las variables sociodemográficas, nuestros resultados no muestran diferencias estadísticamente significativas en la permanencia en IT entre hombres y mujeres. La influencia del sexo en la duración de los periodos de IT ha sido analizada por diferentes autores con resultados discordantes a los nuestros, y así, muchos autores comprueban en sus investigaciones una mayor duración de los episodios de IT en las mujeres respecto a los hombres, seguramente en relación con el uso indebido de la prestación de IT por parte de las mujeres, para cubrir otras deficiencias de nuestro sistema de protección social relacionadas con la conciliación personal y laboral ${ }^{20-23}$.

En relación con la edad los mayores de 60 años presentan una permanencia en IT significativamente mayor a la que presentan los de edades inferiores. Esto no ha sido confirmado en el análisis multivariante. Estos datos coinciden con los presentados en otros estudios ${ }^{24,25}$, y probablemente guardan relación con una mayor morbilidad asociada a la edad y con una mayor dificultad para ejecutar las tareas laborales conforme avanza ésta.

Moncada ${ }^{26}$ mostró que la categoría profesional se relacionaba con la incidencia de episodios de IT, de forma que los trabajadores manuales tenían más incidencia de episodios de incapacidades laborales que los no manuales, haciéndose más evidente ésta diferencia en los episodios de larga duración y sobre todo en el caso de los hombres. Nuestros datos concuerdan en parte con lo anteriormente referido, observándose en nuestra muestra una mayor proporción de trabajadores manuales (sobre todo cualificados, pero también semicualificados y no cualificados) frente a trabajadores no manuales. La menor permanencia en IT observada en los sujetos con mayor nivel de instrucción y en los pertenecientes a las categorías sociales superiores está probablemente relacionada con un acceso más rápido a los tratamientos de los pacientes con mayor poder adquisitivo, lo que les permite una reincorporación a su puesto de trabajo más temprana.

Sólo en el análisis bivariante los trabajadores con un nivel inferior de satisfacción laboral presentan una permanencia en IT significativamente superior respecto a los más satisfechos laboralmente, lo cual es concordante con otros estudios consultados. ${ }^{27}$

La autopercepción de salud mostró, tanto en el análisis bivariante como multivariante, diferencias estadísticamente significativas según el nivel de la misma, indicando una menor permanencia en IT en los sujetos con mejor salud autopercibida. Estos datos 
coinciden con los publicados por otros autores ${ }^{28}$. Son varias las investigaciones que han concluido de forma consistente que la salud autopercibida es una buena medida del estado de salud y se asocia con la mortalidad de la población ${ }^{29}$. Castillo ${ }^{30}$ refiere, además, que la autopercepción de salud es un sencillo y buen indicador de la satisfacción vital, más incluso que el número de enfermedades diagnosticadas, y así los individuos con salud deficiente suelen estar menos satisfechos con sus vidas que los que tienen percepciones más positivas.

El consumo de otros tóxicos, diferentes a alcohol o tabaco, mostró igualmente, tanto en el análisis bivariante como multivariante, diferencias estadísticamente significativas según el consumo o no de dichos tóxicos. El consumo de drogas representa hoy en día uno de los mayores problemas de salud pública en los países industrializados. En los últimos años, lejos de disminuir, se ha ido incrementando progresivamente y, pese a la creencia popular de que el uso abusivo de drogas es mayor en los sectores más desfavorecidos, marginales o con alta tasa de desempleo, no todos los estudios consultados muestran resultados concordantes con esta afirmación. Así, Otero en 2011 mostró que es la población activa quien más consume de forma abusiva dichas drogas ${ }^{31}$. Este consumo conlleva asociada una morbilidad que repercute en el proceso de IT, dificultando la reincorporación del trabajador a su puesto de trabajo.

La permanencia en IT de los sujetos según la naturaleza de su enfermedad, mostró que las enfermedades diferentes a las del aparato locomotor, sistema cardiorrespiratorio y problemas psicológicos se asociaron con una mayor duración del proceso. Las enfermedades de origen neoplásico justifican éstos resultados, siendo este dato concordante con los presentados en otros estudios ${ }^{32}$.

La finalidad del estudio ha sido identificar en qué medida determinados factores influyen en la reincorporación de un paciente a su puesto de trabajo después de permanecer en situación de incapacidad durante un tiempo.

Los resultados de este estudio muestran, mediante análisis de supervivencia, que la permanencia en IT es superior en los trabajadores que padecen enfermedades diferentes a las del aparato locomotor, sistema cardiorrespiratorio o problemas psicológicos, en quienes consumen tóxicos diferentes a alcohol o tabaco y en quienes presentan peor autopercepción de salud o poseen menor nivel de instrucción.

Teniendo en cuenta que en la gestión de la IT, en España y en otros países de nuestro entorno, se han elaborado diferentes manuales y guías cuyos objetivos han sido aproximarse a los tiempos estándar de duración de la IT, la aportación principal de esta investigación es profundizar en el conocimiento de algunos factores que determinan la prolongación de la incapacidad, así como cuantificar el grado en el que dichos factores alargan el proceso de IT. Todo ello es de utilidad en el establecimiento de estándares de duración más objetivos y precisos.

Es posible que los resultados obtenidos sean aplicables a otras áreas de salud de similares características, lo cual podría ayudar a la planificación de programas de intervención enfocados a una mejora en la gestión de la incapacidad temporal.

Recientemente en nuestro país se han emprendido distintas acciones para establecer nuevos modelos de gestión de la IT que permitan optimizar el proceso que genera esta prestación social, la última de ellas viene recogida en el, recientemente publicado, Real Decreto 625/2014 de 18 de julio por el que se regulan determinados aspectos de la gestión y control de los procesos por IT en los primeros 365 días de su duración, y que permite avanzar en la coordinación de todas las entidades que participan en la gestión de esta prestación ${ }^{33}$. Este Real Decreto establece unos protocolos de temporalidad de los actos médicos de confirmación de la baja, que facilitan al médico de atención primaria unos plazos orientativos de control de la prestación, basados en el diagnóstico, la ocupación y la edad del trabajador, al objeto de aligerar los trámites y cargas burocráticas existentes en la actualidad para centrar los procesos de IT en la protección del trabajador 
MEDICINAySEGURIDAD del trabajo !

afectado y en su pronta recuperación, mecanismos que adicionalmente suponen un mejor control de los procesos de IT.

En conclusión, diferentes circunstancias, tanto derivadas de la enfermedad y de la percepción del estado de salud como de las características sociodemográficas o el estilo de vida, intervienen en la reincorporación al ámbito laboral tras la permanencia en incapacidad temporal, lo que corrobora el origen multifactorial de dicha prestación y la necesidad de considerar múltiples aspectos en el análisis de su resolución.

\section{AGRADECIMIENTOS}

Al Instituto Nacional de la Seguridad Social por autorizar el acceso a los datos relativos a los periodos de IT de los pacientes incluidos en este estudio.

\section{REFERENCIAS BIBLIOGRÁFICAS}

1. Morano M. Análisis de la tendencia de la prestación por incapacidad temporal en los presupuestos del sistema de Seguridad Social. Med Segur Trab. 2014; 1:59-64.

2. Monreal I. Vigilancia de la Incapacidad Temporal por Contingencias Comunes. En: La vigilancia de la incapacidad temporal y el actual marco legislativo en salud laboral. Zaragoza: Sociedad de Medicina, Higiene y Seguridad del Trabajo de Aragón y La Rioja; 2007.

3. Álvarez Theurer E, Llergo Muñóz A, Vaquero Abellán M. Análisis de la duración de los periodos de incapacidad temporal por procesos en Andalucia. Factores asociados. Aten Primaria. 2009; 41:387-93.

4. Sánchez-Tabar A, Canga Alonso A, García Cueto E. Absentismo laboral en medio hospitalario. Med Segur Trab. 1989; 36:71-4.

5. Chevalier A, Luce D, Blanc C, Goldberg M. Sickness absence at the French National Electric and Gas Company. BrJ Ind Med. 1987; 44:101-10.

6. Nicholson N, Wall T, Lisheron J. The Predictability of absence and propensity to leave from employees job satisfaction and altitudes toward influence on decisión making. Human Relations. 1977; 30:499-514.

7. Taylor PJ. Causes and control of absenteims. En Encyclopedia of occupational Health and safety. Geneva: International Labour Office. 1983; 4-8.

8. Tsal SP, Dowd CM, Cowles SR, Ross CE. Prospective morbidity surveillance of Shell refinery and petrochemical employees. Br J Ind Med. 1991; 48:155-63.

9. Gawel ZK. Sickness absence of nurses and female doctors in Poland. Int Nurs Rev. 1986; 33:183-5.

10. García-Olmos L, Alonso MT, Pérez de Lucas N, Latorre O. Análisis del absentismo por enfermedad en una empresa de servicios. Una propuesta de coordinación entre los servicios de salud laboral y los profesionales de atención primaria. Rev San Hig Pub. 1990; 64:785-94.

11. Mackeown KD. Sickness absence patterns of 5000 NHS staff employed within Northallerton and South West Durhan Health autorities. J Soc Occup Med. 1987; 37:111-6.

12. Barrallo G, García de Salazar JC, Elola B. Absentismo laboral. Resultado de un estudio realizado en hospital civil de Basurto durante 1983. Estudio comparativo con una entidad bancaria en la provincia de Vizcaya. Gaceta Médica de Bilbao. 1985; 82:59-67.

13. Taylor PJ. Occupational and regional associations of death, disablement, and sickness absence among Post Office Staff 1972-75. Br J Ind Med. 1976; 33:230-5.

14. Benavides FG, Aranaz J, Bolumar F, Alvarez-Dardet C. La incapacidad laboral transitoria, algo más que un problema de salud. Rev San Hig Pub. 1990; 64:749-57.

15. Blanco M, Candelas G, Molina M, Bañares A, Jover JA. Características de la incapacidad temporal de origen músculo-esquelético en la Comunidad de Madrid durante un año. Rev Esp Reuma. 2000; $27: 48-53$.

16. Calvo E. Duración de la incapacidad temporal asociada a diferentes patologías en trabajadores españoles: discusión acerca de la ITCC en los trastornos mentales. Madrid 2010. Disponible en http://www.segsocial.es/prdi00/groups/public/doc uments/binario/146666.pdf.

17. Linares EJ. Los juicios de control sobre los agentes de salud: Variable moduladora de la calidad de vida de los enfermos de cáncer de pulmón avanzado sometidos a tratamiento paliativo. [Tesis Doctoral]. Bellaterra: Universidad Autónoma de Barcelona; 2001. 
Cond trabajo

18. Warr PB, Cook JD, Wall TD. Scales for the measurement of some work attitudes and aspects of psychological well-being. J Occup Organ Psych. 1979; 52:129-48.

19. Goldthorpe J. Sobre la clase de servicio: su formación y su futuro. En: Carabaña J, De Francisco A, editores. Teorías contemporáneas de clases sociales. Madrid: Fundación Pablo Iglesias; 1993. p.229-63.

20. Brage S, Nygard JF, Tellness G. The gender gap in muskuloskeletal related long sickness absence in Norway. Scand J Soc Med. 1998; 26(1):34-43.

21. Steenstra IA, Verbeek JH, Heymans MW, Bongers PM. Pronostic factors for duration of sick leave in patients sick listed with acute low back pain: a systematic review of the literature. Occup Environ Med. 2005; 62:851-60.

22. Joiling C, Groot W, Jansen P. Duration dependence in sickness absence: how can we optimize disability management intervention strategies? J Occup Environ Med. 2006; 48:803-14.

23. Benavides FG. Informe de salud laboral en España 2006. Barcelona: Observatorio de salud laboral; 2007.

24. Krause N, Frank JW, Dasinger LK, et al. Determinants of duration of disability and return-to-work after work-related injuri and illness: Challenges for future research. Am J Ind Med. 2001; 40:464-84.

25. Torá I, Martínez JM, Delclós J, Jardí J, Alberti C, Serra C, Manzanera R, Benavides FG. Duración de los episodios de incapacidad temporal por contingencia común según regiones sanitarias en Catalunya. Rev Esp Salud Pública. 2010; 84:61-69.

26. Moncada S. Continguts del treball i incapacitat temporal en la cohort «Casa Gran» de treballadors de L'Ajuntament de Barcelona [Tesis Doctoral]. Barcelona: Universitat Autònoma de Barcelona; 2000.

27. Araña M, Laborda E, Cámara R. Regreso al trabajo. Una intervención bio-psico-jurídico-social estratégicanecesaria para la resolución de la Incapacidad Laboral Temporal por problemas de salud mental. Madrid: Ministerio de Trabajo e Inmigración; 2008.

28. Vaquero M. Factores que influyen en la incapacidad temporal de larga duración: propuesta de screening y de intervención. Disponible en http://www.seg-social.es/prdi00/groups/public/ documents/binario/168544.pdf.

29. Jylhä M. What is self-rated health and why does it predict mortality? Towards a unified conceptual model. Soc Sci Med. 2009; 69:307-16.

30. Castillo A, Albala C, Dangour A, Uauy R. Factores asociados a satisfacción vital en una cohorte de adultos mayores de Santiago de Chile. Gac Sanit. 2012; 26(5):414-20.

31. Otero C. Drogodependencias en el lugar de trabajo. Pautas generales de intervención desde la medicina del trabajo. Med Segur Trab. 2011; 57(1):1-262.

32. Delclós J, Gimeno D, Torá I, Martínez JM, Manzanera R, Jardí J, et al. Distribución de la duración de la incapacidad temporal por contingencia común por diagnóstico médico (Cataluña, 2006-2008). Gac Sanit. 2013; 27(1):81-83.

33. Real Decreto $625 / 2014$, de 18 de julio, por el que se regulan determinados aspectos de la gestión y control de los procesos por incapacidad temporal en los primeros trescientos sesenta y cinco días de su duración. BOE n. ${ }^{\circ}$ 176, de 21 de julio de 2014.

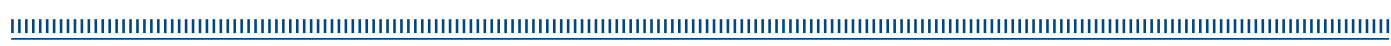

